\title{
Both the V2 and V3 Regions of the Human Immunodeficiency Virus Type 1 Surface Glycoprotein Functionally Interact with Other Envelope Regions in Syncytium Formation
}

\author{
ARNO C. ANDEWEG, PAULA LEEFLANG, ALBERT D. M. E. OSTERHAUS, \\ AND MARNIX L. BOSCH* \\ Laboratory of Immunobiology, National Institute of Public Health and \\ Environmental Protection, Bilthoven, The Netherlands
}

Received 3 September 1992/Accepted 9 March 1993

\begin{abstract}
To map the regions of the external envelope glycoproteins of human immunodeficiency virus type 1 (HIV-1) involved in the process of membrane fusion, we determined the syncytium-inducing capacity of a panel of transiently expressed chimeric envelope genes. This panel was generated by exchanging gene fragments between four previously studied envelope genes that exhibited a high degree of sequence homology yet displayed marked differences in syncytium-inducing capacity when expressed by recombinant vaccinia virus. The results demonstrate that multiple regions of the HIV-1 envelope glycoproteins are involved in syncytium formation. Some fragments, most notably those containing the V2 or V3 region, can transfer syncytium-inducing capacity to envelope proteins previously not capable of inducing syncytia. Moreover, it is shown that such regions functionally interact with other envelope regions, especially one encompassing the V4 and V5 regions of gp120 or a region encompassing part of gp41, to exert their function in membrane fusion.
\end{abstract}

Human immunodeficiency virus type 1 (HIV-1) enters the cell through a multistep process. Binding of the viral external glycoprotein gp120 to the cellular receptor CD4 ultimately results in fusion of the viral envelope and the cellular membrane $(6,22)$. A similar fusion process between HIV-1infected cells expressing viral envelope glycoproteins and (uninfected) cells expressing CD4 may lead to the formation of syncytia $(23,29)$.

HIV-1 isolates differ in their capacity to induce syncytia in vitro. On the basis of both this capacity and other in vitro biological properties, such as replication rate and cytotropism, syncytium-inducing (SI) and non-SI (NSI) HIV-1 isolates are distinguished (35). These two categories largely overlap with the "rapid-high" and "slow-low" viruses, respectively, described by others (10). The isolation of HIV-1 variants with an SI phenotype correlates with the subsequent rapid decline of $\mathrm{CD}^{+}$cell numbers and progression to AIDS $(4,21,36)$, which suggests that the emergence of viruses with a cytopathic SI phenotype plays a role in the pathogenesis of AIDS. Dissection of the process of membrane fusion that leads to syncytium formation may contribute to our understanding of its role in HIV-1 biology and pathogenesis.

HIV-1 envelope glycoproteins contain all of the viral information required for syncytium formation $(22,34)$, and several of the determinants that mediate the underlying membrane fusion process have been identified. Genetic variation in such determinants could theoretically result in differences in SI capacity between different HIV-1 envelope genes. For efficient binding to the cellular receptor CD4, an intact CD4-binding region is needed. Amino acid residues involved in CD4 binding are scattered among at least four different regions of gp120 (26). The potential effects of variation in these regions on syncytium formation has not yet been studied in detail. Syncytium formation by both

\footnotetext{
* Corresponding author.
}

simian immunodeficiency virus and HIV-1 envelope genes could be experimentally manipulated by introducing mutations in the fusion domain at the amino terminus of the transmembrane glycoprotein gp41 $(2,12)$. Although natural sequence variation in the fusion domain of SI and NSI isolates was also found, such variation does not contribute significantly to the differences in syncytium formation (1). Naturally occurring sequence variation in the V3 loop, however, does seem to be relevant for the differences in the capacities of HIV-1 isolates to induce syncytia. Site-directed mutagenesis experiments have demonstrated a direct role for this region in HIV-1-induced membrane fusion $(13,27)$, and genetic exchange experiments between molecular clones of HIV-1 with different SI capacities have shown that such differences may map to the $\mathrm{V} 3$ region $(5,8)$. This same region also contains determinants that control HIV-1 cell tropism (20). Taken together, these data suggest a pivotal role for the V3 region of HIV-1 envelope.

Neutralizing antibodies directed against both the CD4binding region and the V3 region have been described previously $(16,17,28,30,32,37)$. Such antibodies exert their function upon binding to their target sites either by inhibition of CD4 binding or through interference with an essential step in the process that leads from CD4 binding to fusion. Neutralizing antibodies that are directed against envelope regions for which no function in the membrane fusion process is known (e.g., the V2 region [14] or the central region of gp41 [7]) have also been described. These data and the results of studies employing site-directed mutagenesis $(19,38)$ suggest that other regions than those described above may be involved in the membrane fusion process. Nevertheless, in studies in which gene fragments are exchanged between pairs of molecular clones with different SI capacities, the determinants involved are repeatedly mapped to an envelope gene fragment that always includes the V3 region that has already been identified $(5,8)$. In an attempt to screen for additional membrane fusion-mediating determi- 


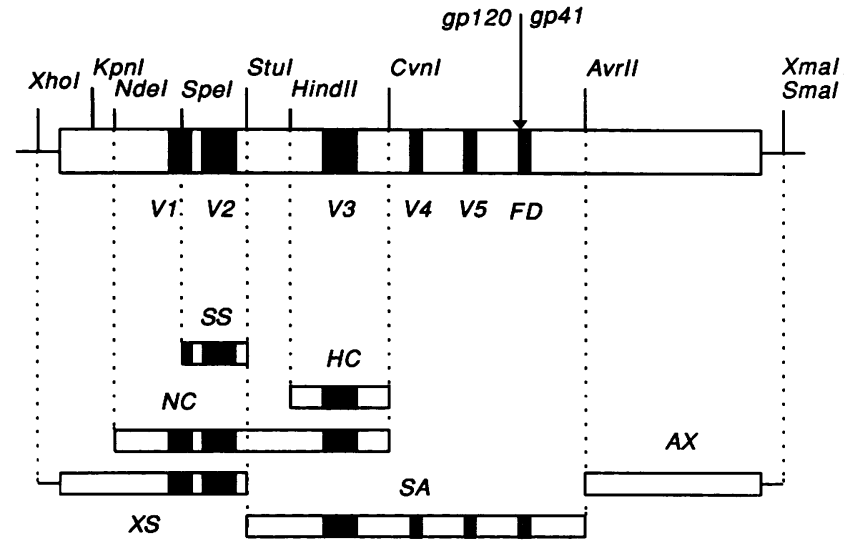

FIG. 1. Positions of the generated gene fragments relative to the complete envelope gene (top bar). The hypervariable regions V1 to V5, fusion domain (FD), cleavage site (arrow), and the restriction enzymes used are shown. The gene fragments are named after the flanking restriction sites.

nants, we performed the mapping experiments described in this report.

The starting material for this study consisted of four complete HIV-1 envelope genes described elsewhere (1). Since these envelope genes were obtained from one patient at a single time point, these genes are highly related genetically, yet they display heterogeneous SI capacities: two induce syncytia in peripheral blood mononuclear cells and in SUP-T1 cells, whereas the two others do not. We have generated 40 chimeric envelope genes, mainly between SI and NSI envelope genes, and tested these genes in a syncytium-forming assay. Our results demonstrate that multiple regions of the HIV-1 envelope glycoproteins control SI capacity; most notably, those regions that contain the V2 or V3 region can transfer SI capacity to envelope proteins previously not capable of inducing syncytia. Moreover, our data show that the capacity of the V2 and V3 regions to mediate syncytium formation also depends on additional envelope regions encompassing the V4 and V5 regions of gp120 or a region encompassing part of gp41.

\section{MATERIALS AND METHODS}

Construction of chimeric envelope genes. Four previously characterized HIV-1 envelope genes from biological clones obtained from patient 16 at a single time point and designated 16.1 to 16.4 (1) were used to generate a set of chimeric envelope genes. These parental genes were originally cloned in the pSC11 vaccinia virus expression vector (3) and in the plasmid pGEM7- (Promega). Chimeric envelope genes were constructed by exchanging gene fragments generated with restriction enzymes (NdeI, SpeI, StuI, HindII, CvnI, and $A v r I I)$ with conserved restriction sites in all four envelope genes and the restriction enzymes $X h o I$ and $X m a I$, which provide convenient sites in the cloning vectors, as depicted in Fig. 1. The resulting envelope chimeras are designated according to the exchanged gene fragment and the two parental envelope genes (see Fig. 3). All chimeric envelope genes were tested by restriction mapping using restriction sites that were not conserved in the original four envelope genes. Occasionally, chimeric envelope genes were partially sequenced to verify the cloning procedure.

Cloning of chimeric envelope genes in transient expression vector. The chimeric envelope genes were cloned in the simian virus 40-based expression plasmid pSRHS (a generous gift of Eric Hunter, University of Alabama at Birmingham, Birmingham [9]). The HIV env gene sequences were cloned by exchanging the original env gene of HXB2 with KpnI and SmaI restriction enzymes (Fig. 1). All the expressed chimeric envelope genes thus contain the signal peptide and the first 13 amino acids of the HXB2 envelope protein.

DNA transfection and syncytium formation assay. All generated constructs were transfected in CEMx174 cells (31) by electroporation. Five million cells were mixed with $20 \mu \mathrm{g}$ of DNA in $250 \mu$ l of RPMI medium (GIBCO) supplemented with $10 \%$ fetal calf serum (FCS) in a 4-mm-wide electroporation cuvette (Eurogentec S.A.). After electroporation (200 $\mathrm{V}, 960 \mu \mathrm{F}$; Bio-Rad gene pulser), the cells were kept on ice for $10 \mathrm{~min}$ and then transferred to small culture flasks containing $10 \mathrm{ml}$ of RPMI medium. Forty-eight hours after electroporation, viable cells were isolated on a Ficoll density gradient, and $2 \times 10^{5}$ of these cells were cocultured for $16 \mathrm{~h}$ with an equal amount of SUP-T1 cells (33). Syncytia were only found after cocultivation. The relative sizes and numbers of syncytia found in each culture were scored independently by three researchers. The size of syncytia was quantified as follows: small $(+)$, up to five times the size of single cells; large $(+++)$, giant syncytia estimated to contain over 100 fused cells (scored as being larger than $250 \mu \mathrm{m}$ in diameter), or intermediate $(++)$. For examples of syncytium size, see Fig. 2.

env expression. At the time that the cultures were scored for syncytium formation, separately cultured portions of the Ficoll-purified cells were treated as follows: (i) spotted onto microscope slides and fixed with ice-cold ethanol (70\%) for immunofluorescence and stored at $-20^{\circ} \mathrm{C}$ and (ii) lysed with $1 \%$ empigen BB (25) and frozen at $-70^{\circ} \mathrm{C}$ for enzyme-linked immunosorbent assay. Finally, a sample of the culture supernatants was frozen and also tested in an ELISA.

(i) Immunofluorescence. The percentage of envelope protein-expressing cells was determined by an immunofluorescence assay. The fixed cells on microscope slides were incubated with patient serum (1:70 dilution) and exposed to fluorescein isothiocyanate (FITC)-conjugated sheep antihuman immunoglobulin G1. The percentages of positive cells were scored with a fluorescence microscope.

(ii) gp120 ELISA. To determine the relative amounts of envelope protein produced in the different transfected cell cultures, a gp120 ELISA that is based on an assay developed by Moore et al. $(24,25)$ was used. Capture antibodies D7324 (Aalto BioReagents, Dublin, Ireland) were adsorbed on to ELISA plates (Costar) by incubation overnight in $40 \mathrm{mM}$ $\mathrm{NaHCO}_{3}, \mathrm{pH} 9.6$, at $4^{\circ} \mathrm{C}$. D7324 is an affinity-purified polyclonal sheep antiserum to the conserved carboxy-terminal 15 amino acids of gp120. The predicted amino acid sequences of the four envelope genes used in this study are completely homologous to this peptide (1). Unbound antibodies were removed by washing each well four times with $200 \mu \mathrm{l}$ of the assay buffer $(0.1 \mathrm{M} \mathrm{NaCl}, 0.1 \mathrm{M}$ Tris, $0.1 \%$ Tween 20, pH 7.5). All incubations were performed for $1 \mathrm{~h}$ at $37^{\circ} \mathrm{C}$ with assay buffer supplemented with $1 \%$ FCS and $0.5 \%$ normal goat serum and terminated by washing each well four times with $200 \mu$ l of assay buffer unless stated otherwise. The wells were subsequently blocked with assay buffer supplemented with serum. Next the wells were incubated with $100-\mu l$ portions of a serial dilution of cell lysate or culture supernatant for $2 \mathrm{~h}$. Detection of the attached gp120 was achieved by successive incubation of the wells with the 

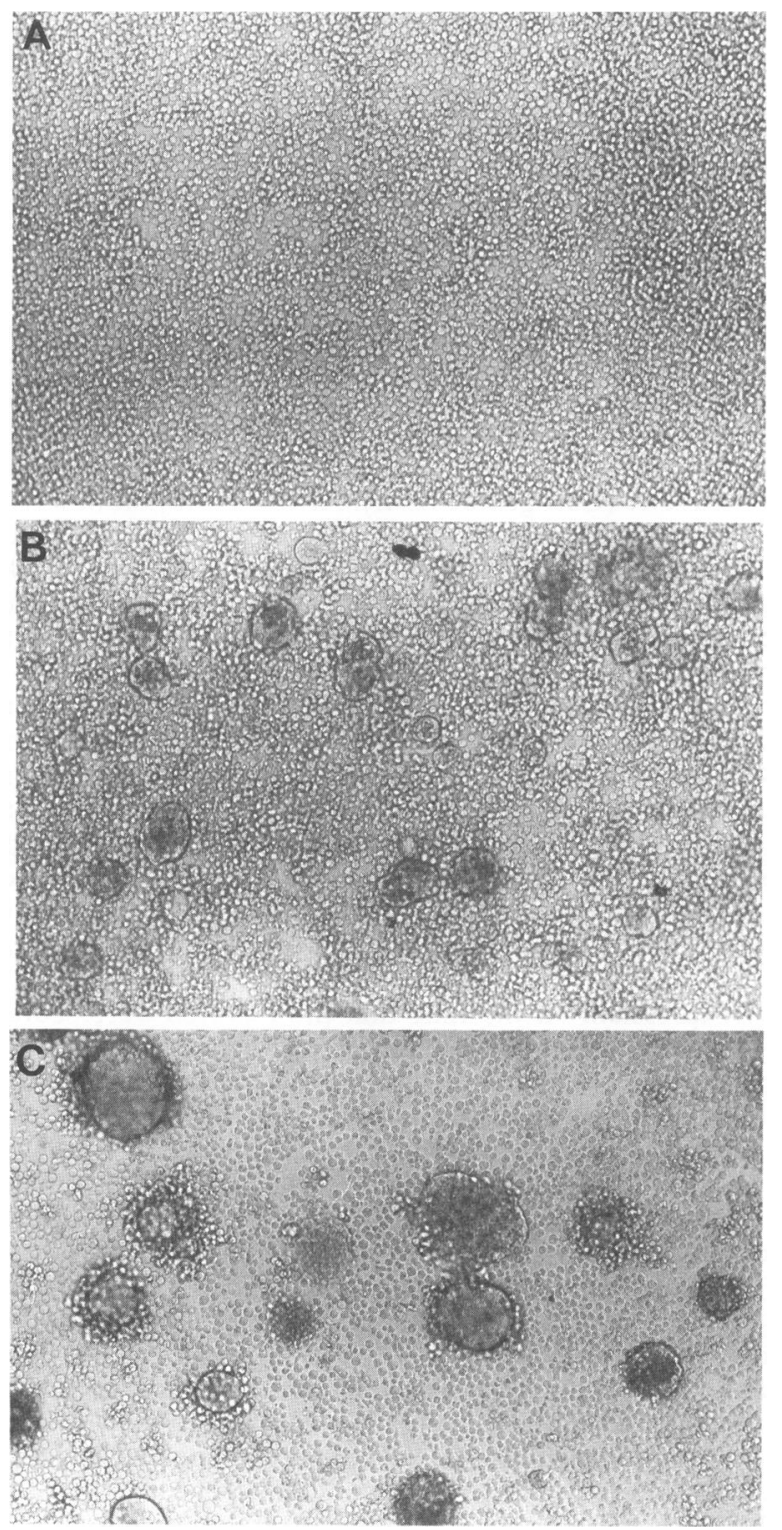

FIG. 2. Syncytia induced upon cocultivation of transfected CEMx174 cells with SUP-T1 cells. (A) No syncytia; intermediatesize syncytia (B); and large syncytia (C).

following: a 1:2,000 dilution of patient serum, a 1:2,000 dilution of biotinylated goat antihuman IgG (Amersham), and streptavidin-horseradish peroxidase conjugate (streptABComplex/HRP [Dako A/S]). Finally, the plates were incubated with tetramethylbenzidine $-\mathrm{H}_{2} \mathrm{O}_{2}$. The reaction was stopped by adding $50 \mu \mathrm{l}$ of $2 \mathrm{M} \mathrm{H}_{2} \mathrm{SO}_{4}$, and the optical density at $450 \mathrm{~nm}$ was read with a Titertek Multiskan (Flow Laboratories). All gp120 ELISA values for the different constructs were related to each other and expressed as a percentage of the maximum value measured. The assay can routinely detect $20 \mathrm{pg}$ of recombinant gp120 (Celltech, provided to us through the Medical Research Council AIDS reagent project).

(iii) CD4 ELISA. The CD4 ELISA used was very similar to the gp120 ELISA described above. The only difference was that the envelope proteins are now captured to the solid phase by binding to attached CD4. Recombinant soluble CD4 (100 ng/well; American Bio-Technologies; provided to us through the Medical Research Council AIDS reagent project) was adsorbed on to ELISA plates (Costar) by incubation overnight in phosphate-buffered saline ( $\mathrm{pH} \mathrm{7.2)} \mathrm{at}$ $4^{\circ} \mathrm{C}$. All subsequent incubations were performed as described for the gp120 ELISA, except that the detecting patient serum was diluted 1:3,000.

Nucleotide sequence accession numbers. The GenBank accession numbers for the envelope gene sequences derived from biological clones 16.1 to 16.4 are $\mathrm{L} 08655$ to L08658, respectively.

\section{RESULTS}

Construction of chimeric envelope genes. Chimeric envelope genes were constructed from the four parental envelope genes $16.1,16.2,16.3$, and 16.4 described elsewhere (1). We exchanged six different fragments between these four parental envelope genes to generate the 40 chimeric envelope genes (Fig. 1 and 3 ). The chimeric envelope genes were expressed in CEMx174 cells in transient expression experiments.

Expression of gp120. Expression of the envelope protein in the transfected cells was monitored by ELISA and immunofluorescence. In Table 1 , the relative amount of gp120 produced (cell associated and shed in the culture medium) is listed for each construct tested. The percentage of HIV-1 envelope protein-expressing cells in the population of transfected CEMx174 cells varied from 0.6 to $4.6 \%$ of the total (Table 1).

CD4 binding of expressed gp120. By immunoprecipitation we have previously shown that all four parental envelope gene products were able to bind soluble CD4 (1). For the present study, we also tested the expressed chimeric envelope proteins for their ability to bind CD4. All chimeric envelope proteins produced upon transfection of the generated constructs were able to bind CD4, as demonstrated by the CD4-binding ELISA (results not shown). The exchange of envelope gene fragments apparently did not interfere with the CD4-binding abilities of these expressed gene products.

SI capacities of expressed envelope genes. In a previous study (1), we have shown that upon expression in a recombinant vaccinia virus, the two parental SI envelope gene products 16.1 and 16.2 induce syncytia in SUP-T1 cells, whereas the NSI envelope gene products do not. For the experiments described here, we therefore used SUP-T1 cells as CD4-positive fusion partners. Upon transfection of CEMx174 cells and subsequent cocultivation with SUP-T1 cells, almost all expressed envelope chimeras gave rise to the formation of syncytia. The sizes of the induced syncytia differed substantially for the different constructs and were highly reproducible. We have defined the SI capacity of the chimeric envelope gene products as the maximum size of the syncytia they induce in SUP-T1 cells. Within any range of the quantitative parameters shown in Table 1 , small and large syncytia were observed. These results indicate that syncytium size is a qualitative property of the (chimeric) envelope and that the syncytium size is not dependent on experimental variables that influence the amount of envelope proteins produced at least not within the range observed in these experiments.

Figure 3 shows the SI capacities of all generated chimeric envelope constructs. The data presented are derived from single representative experiments. Several control experiments were performed, including multiple testing of the 
TABLE 1. Quantitative expression of gp120 by (chimeric) envelope genes and sizes of the induced syncytia

\begin{tabular}{|c|c|c|c|}
\hline $\begin{array}{c}e n v \\
\operatorname{code}^{a}\end{array}$ & $\begin{array}{c}\text { Syncytium } \\
\text { size }^{b}\end{array}$ & $\begin{array}{l}\text { Envelope protein- } \\
\text { expressing cells } \\
\text { by IFA }(\%)^{c}\end{array}$ & $\begin{array}{c}\operatorname{gp} 120 \\
\text { ELISA }(\%)^{d}\end{array}$ \\
\hline 16.1 & +++ & 2.6 & 18 \\
\hline 16.2 & +++ & 1.9 & 42 \\
\hline 16.3 & - & 3.8 & 36 \\
\hline 16.4 & - & 2.7 & 27 \\
\hline $12 \mathrm{XS}$ & ++ & NT & 76 \\
\hline $21 X S$ & +++ & 1.9 & 23 \\
\hline $12 \mathrm{NC}$ & +++ & 1.7 & 17 \\
\hline $21 \mathrm{NC}$ & +++ & 3.4 & 23 \\
\hline $12 \mathrm{HC}$ & +++ & 4.1 & 45 \\
\hline $12 \mathrm{AX}$ & +++ & 2.2 & 23 \\
\hline $21 \mathrm{AX}$ & +++ & 2.8 & 20 \\
\hline $13 \mathrm{XS}$ & ++ & 3.3 & 36 \\
\hline $14 \mathrm{XS}$ & ++ & 1.3 & 27 \\
\hline 13SS & ++ & NT & 52 \\
\hline $14 S S$ & ++ & 2.0 & 22 \\
\hline 31SA & +++ & 0.6 & 38 \\
\hline $31 \mathrm{AX}$ & +++ & 1.0 & 67 \\
\hline $31 \mathrm{HC}$ & +++ & 4.6 & 100 \\
\hline $14 \mathrm{NC}$ & +++ & 2.9 & 25 \\
\hline 13SA & +++ & 1.6 & 77 \\
\hline 14SA & +++ & 2.3 & 25 \\
\hline $31 X S$ & +++ & 1.8 & 45 \\
\hline $41 X S$ & +++ & 1.8 & 19 \\
\hline $13 \mathrm{HC}$ & +++ & 2.7 & 28 \\
\hline $14 \mathrm{HC}$ & +++ & 4.2 & 39 \\
\hline $13 \mathrm{AX}$ & + & 0.9 & 54 \\
\hline $31 \mathrm{NC}$ & ++ & 3.7 & 58 \\
\hline 41NC & + & 3.2 & 69 \\
\hline $24 X S$ & ++ & 1.8 & 29 \\
\hline $23 X S$ & + & 1.2 & 44 \\
\hline $42 \mathrm{SA}$ & ++ & 1.5 & 38 \\
\hline $23 \mathrm{HC}$ & ++ & NT & 30 \\
\hline $23 \mathrm{NC}$ & + & 2.6 & 26 \\
\hline $42 \mathrm{NC}$ & + & 1.9 & 31 \\
\hline $32 \mathrm{NC}$ & ++ & 3.3 & 26 \\
\hline $24 \mathrm{AX}$ & - & 1.5 & 29 \\
\hline $42 \mathrm{AX}$ & +++ & 1.8 & 45 \\
\hline $32 \mathrm{AX}$ & +++ & 1.2 & 18 \\
\hline $42 \mathrm{HC}$ & +++ & 1.8 & 42 \\
\hline $42 \mathrm{SSCX}$ & +++ & 1.5 & 25 \\
\hline $32 \mathrm{HC}$ & +++ & 3.3 & 50 \\
\hline 24SA & +++ & 1.3 & 34 \\
\hline 23SA & +++ & 4.6 & 31 \\
\hline $32 \mathrm{XS}$ & +++ & 1.9 & 80 \\
\hline
\end{tabular}

${ }^{a}$ Chimeric genes were named so that the name reflects construction of the chimeric gene. $A B C D$ is a chimeric envelope gene in which gene fragment $C D$ from parental env gene A is transferred to gene B. The envelope genes obtained from biological clones 16.1 to 16.4 are referred to as 1 to 4 .

${ }^{b}$ SI capacity of expressed envelope genes. Symbols: -, no syncytia; +, small syncytia; ++ , intermediate-size syncytia; +++ , large syncytia.

c Percentage of envelope protein-expressing CEMx174 cells as monitored in an immunofluorescence assay (IFA). NT, not tested.

${ }^{d}$ Relative amount of gp120 produced by transfected CEMx174 cells (shed in culture medium and cell associated) expressed as a percentage of the maximum observed gp120 expression. same construct and complete reconstruction of some envelope chimeras. In all of these cases, essentially similar results were obtained.

Mapping of envelope determinants controlling SI capacity. Figure 3A first shows the four parental envelope genes with the sizes of the induced syncytia in SUP-T1 cells. As in our experiments in which these same envelope genes were expressed by recombinant vaccinia virus, only SI clonederived envelope gene products were capable of inducing (large) syncytia (1). The same panel also lists seven chimeric envelope constructs in which gene fragments between the two SI clone-derived envelope genes (16.1 and 16.2) have been exchanged. With the exception of construct 12XS that induces intermediate-size syncytia, all constructs consisting of solely SI clone-derived envelope gene sequences were able to induce large syncytia.

For further analysis, we divided our constructs in two subsets that each had sequences of one of the parental SI envelope genes as a common denominator. Figure $3 \mathrm{~B}$ and $\mathrm{C}$ summarize the data for the subsets based on the SI genes 16.1 and 16.2 , respectively, which were expected to yield information on regions that positively influence syncytium formation. Interestingly, dissimilar results were obtained for both subsets.

Analysis of the constructs based on 16.1 sequences revealed two small distinct regions containing determinants that are important in syncytium formation. These regions are the SS fragment located in the amino-terminal part of gp120 (Fig. 3B, top) and the central HC fragment (Fig. 3B, center). Both small fragments transfer considerable SI capacity from the 16.1 SI envelope to both NSI envelope backgrounds. The 16.1 HC fragment alone transforms the NSI envelopes into full SI envelopes upon expression. The 16.1 SS fragment (or the larger XS fragment of 16.1) by itself transfers only the capacity to form medium-size syncytia but in the presence of other 16.1-derived fragments can transfer full SI capacity (Fig. 3B, top).

Chimeric envelopes based on 16.2 sequences show also that the same two distinct envelope regions are important in syncytium formation. However, like the SS region of 16.1 , now both regions transfer full SI capacity only in the presence of additional SI-derived sequences in the same chimeric envelope construct (Fig. 3C).

\section{DISCUSSION}

We are studying the process of syncytium formation as a model system to unravel the underlying mechanism of HIV1 -induced membrane fusion. In the present study, we have mapped the regions of the HIV-1 envelope proteins which determine the SI capacity.

It has been demonstrated that syncytium formation is mediated by the envelope glycoproteins of HIV-1, although the exact mechanism has not been clarified $(22,34)$. In a previous study (1), we provided evidence that differences in the SI capacities of field isolates are dependent on differences in the envelope gene itself. To avoid possible interference of long terminal repeat or regulatory gene functions with envelope-induced syncytium formation, we have chosen to analyze chimeric envelope genes that are transiently expressed out of the context of the viral genome. The highly homologous HIV-1 envelope genes that were obtained from a single donor at the same time point and that were previously analyzed genetically and functionally served as the basis for the experiments described here.

Since transient expression experiments lack internal con- 

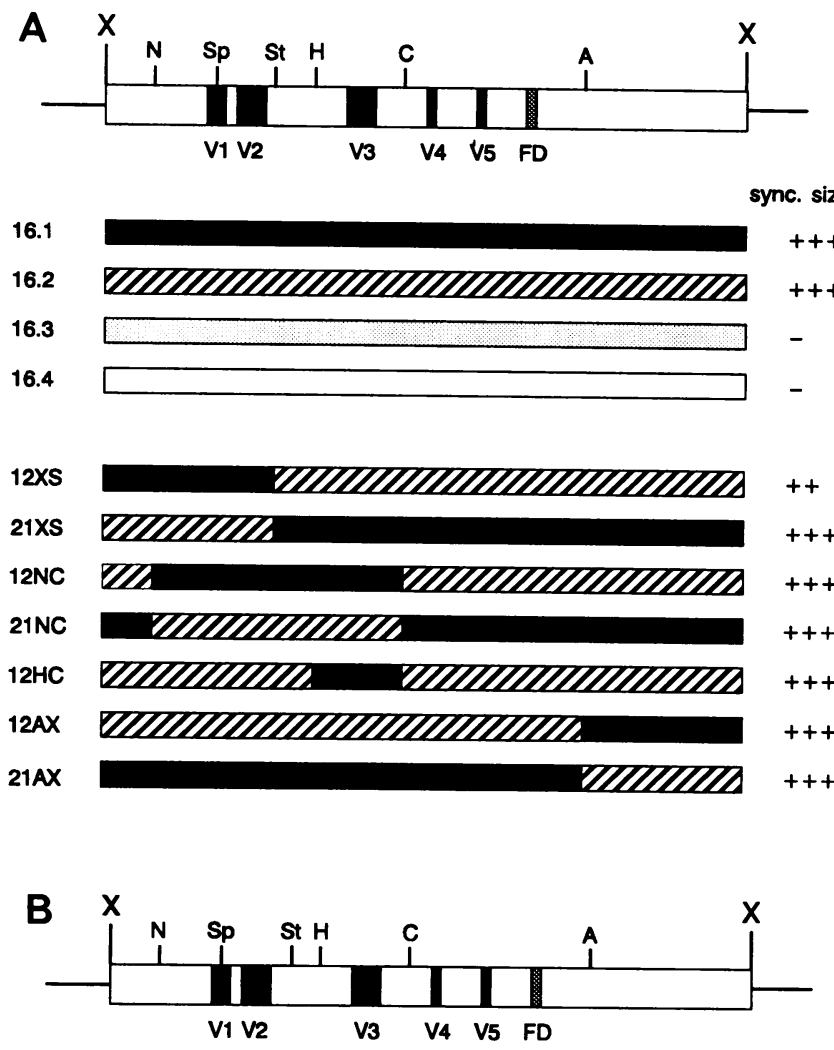

sync. size

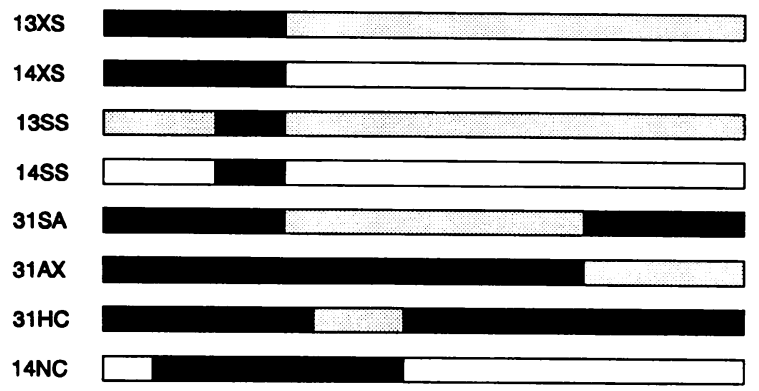

$+$

$++$

$++$

$++$

$+++$

$+++$

$+++$

$+++$

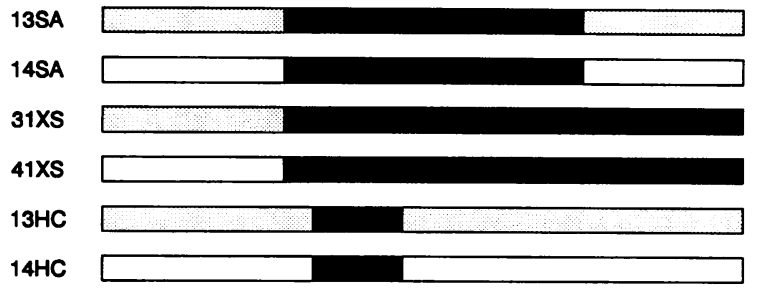

$+++$

$++$

$+++$

$+++$

$+++$

$+++$

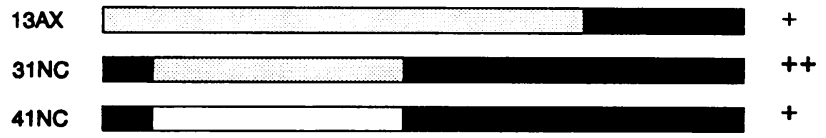

trols for protein expression and function, we first studied the levels of envelope protein production and the CD4-binding capacity. For each transfection, experimental variables, such as the percentage of gp120-producing cells, the relative amount of gp120 produced, the number of syncytia, and the
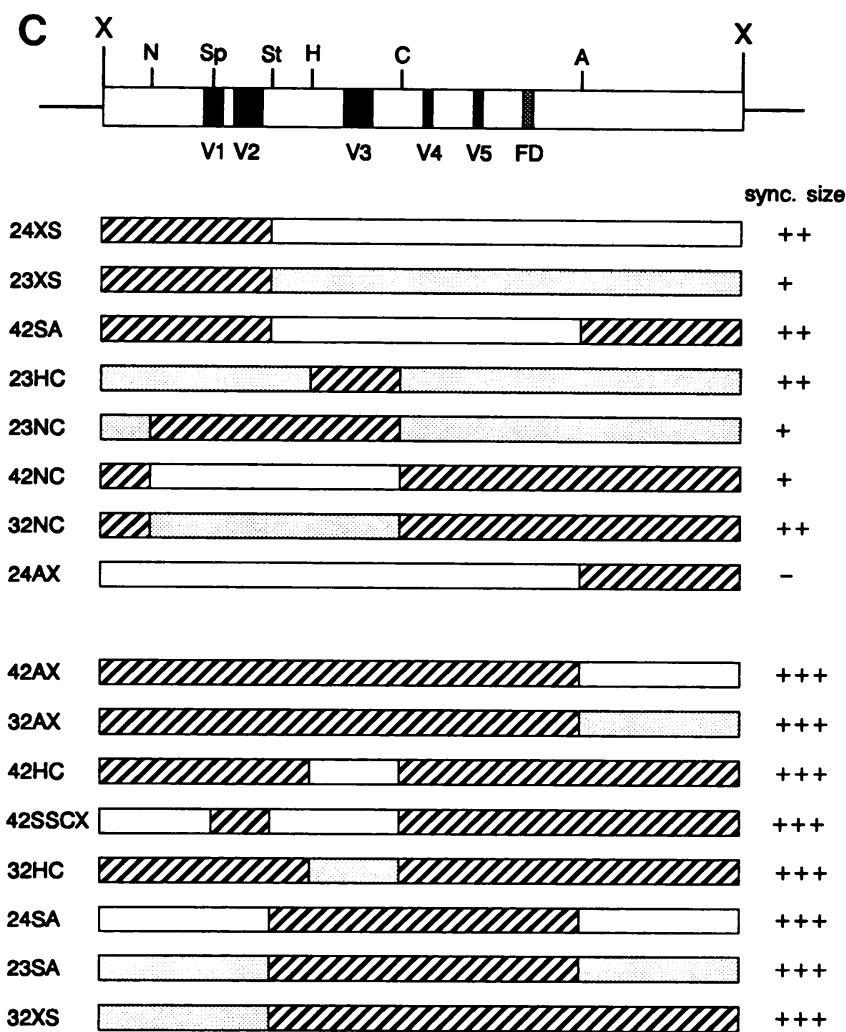

FIG. 3. Syncytium size induced by (chimeric) envelope gene products. (A) The syncytium sizes of the expressed parental envelope genes (16.1 to 16.4) which were used to generate the chimeric envelope genes and chimeric envelope gene products based on solely SI clone-derived sequences (16.1 and 16.2). (B and C) Syncytium sizes of chimeric envelope genes based on envelope gene SI 16.1 (B) or SI 16.2 (C). At the top of each panel, the complete envelope gene is represented by a bar and the restriction enzymes used to exchange gene fragments are indicated. Abbreviations: FD, fusion domain; X (left end), XhoI; N, NdeI; Sp, SpeI; St, StuI; H, HindII; C, CvnI; A, AvrII; X (right end), XmaI; sync., syncytium. Symbols: + , small syncytia; ++ , intermediate-size syncytia; +++ , giant syncytia; -, no syncytia (for more details, see Materials and Methods).

CD4-binding capacity of the expressed recombinant protein, were monitored. Within any range of the quantitative parameters, small and large syncytia were observed, which excluded the possibility that envelope protein expression was a limiting factor in our experiments. This notion was supported by the observation that none of the quantitative parameters correlated with the readouts of the experiments; the size of the induced syncytia appears to be a genetic trait of each individual construct. Finally, all expressed (chimeric) envelope proteins were able to bind CD4.

In our approach, we have transferred fragments of SI envelope genes to NSI envelope genes searching for regions that positively influence the SI capacity. All of these constructs therefore contain genetic information from both SI and NSI envelope genes. We found no evidence for NSI clone-derived envelope regions that negatively influence the SI capacity, but we were able to identify regions from SI genes that transfer SI capacity to NSI backgrounds. The mapping experiments show that multiple determinants located on several envelope gene fragments contribute to syncytium formation. In our opinion, the effects described 


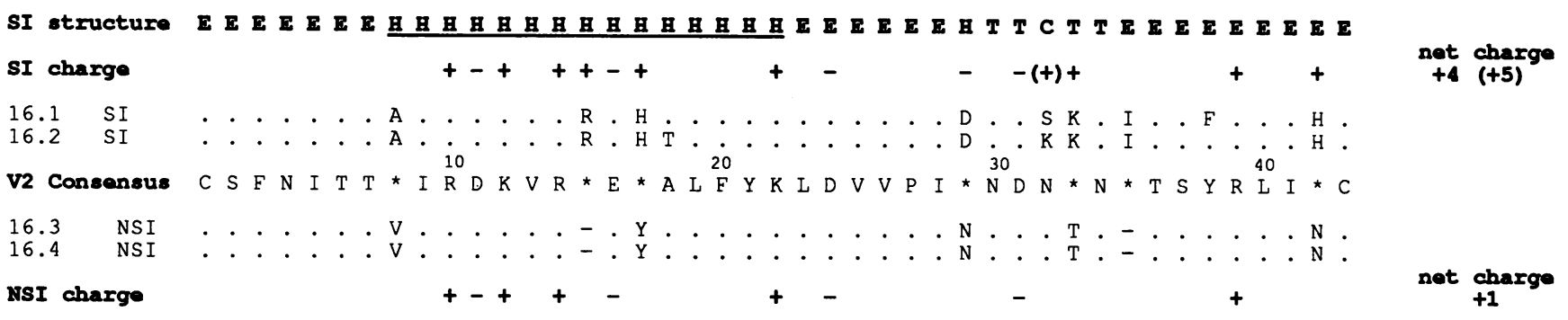

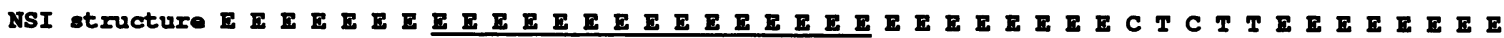

FIG. 4. Predicted amino acid sequences of the hypervariable region V2 together with the predicted secondary structures and charge differences. Amino acids identical to those of the consensus sequence (.) and deletions ( - ) are indicated. The predicted secondary structures of the SI and NSI gene products are also given, at the top and bottom of the figure. The corresponding amino acid is predicted to be in the following configurations; helical $(\mathrm{H})$, extended stretch $(\mathrm{E})$, turn $(\mathrm{T})$ and coil $(\mathrm{C})$. The underlined regions are predicted to have significantly different secondary structures for the SI and NSI clone-derived V2 amino acid sequences. The charges are given in boldface type.

here are the results of multiple functional interactions between different regions of the envelope glycoproteins, we distinguish SI regions (SIRs), regions which are by themselves capable of transferring considerable SI capacity, and enhancing regions that enhance the SI capacity of SIRs but by themselves are not or hardly capable to induce syncytia. Both the SS and HC gene fragments of the 16.1 and 16.2 SI envelopes can independently transfer considerable SI capacity to an NSI envelope which identifies these regions as SIRs. The $16.1 \mathrm{HC}$ fragment alone is sufficient to transfer full SI capacity. This SIR apparently functions independently of the background sequence into which it is cloned. For the other identified SIRs (the 16.2 HC fragment and the SS fragment of 16.1 and 16.2) function is context dependent: their SI capacity can be increased by the simultaneous transfer of an enhancing region. An alternative explanation for the described enhancements of syncytium formation by enhancing regions could be a purely additive effect, since the enhancing regions by themselves can also transfer some SI capacity to NSI gene products. However, such additive effects are not seen when two SIRs, e.g., the V2 and V3 regions of 16.2 , are combined in one construct in the absence of an enhancing region (construct $23 \mathrm{NC}$ [Fig. $3 \mathrm{C}$ ]). It is therefore more likely that the effects observed upon transfer of enhancing regions from SI genes to NSI genes result from the "activation" of cryptic SIRs present in the NSI background through the functional interactions postulated here.

The SIR-containing HC fragment codes for 107 amino acids and encompasses the hypervariable region V3, which suggests that the V3 loop region is a major determinant for syncytium formation for the envelope genes studied here. The SI clone-derived V3 loops (16.1 and 16.2) share a positively charged amino acid (arginine) at position 25 of the V3 loop, whereas the NSI clone-derived V3 loops (16.3 and 16.4) both have a negatively charged glutamic acid at this position. This same phenotype-correlated amino acid variation has also been found in a large set of V3 loop sequences (11). The four parental genes, however, also showed considerable amino acid variation just $C$ terminal of the V3 loop itself. This region, which is present in the small $\mathrm{HC}$ fragment, also contains phenotype-associated amino acid variations, e.g., the SI genes have a positively charged residue (lysine) at position 351, where the NSI genes have a negatively charged glutamic acid residue (1). Additional experiments to demonstrate exactly which residues on the HC fragment are responsible for the observed transfer of SI capacity are needed.
Interestingly, the extremely small SI gene-derived SS fragment alone is also capable of transferring SI capacity to an NSI gene product. This SS fragment, coding for only 71 amino acids, encompasses part of the variable region $\mathrm{V} 1$ and the complete variable region V2. The V1 region is identical for all four parental envelope proteins, and all amino acid variation of the SS fragment is confined to the V2 region. Constructs $24 \mathrm{XS}, 42 \mathrm{HC}, 32 \mathrm{HC}$, and $42 \mathrm{SSCX}$, all based on 16.2 SI envelope sequences, demonstrate that the $\mathrm{V} 2$ region is also an important determinant in syncytium formation for the 16.2 envelope. Examination of the amino acid variation of the V2 region revealed that the SI clone-derived V2 sequences differ from the NSI clone-derived sequences in charge and predicted secondary structure. The variable amino acids of the SI V2 sequences are often positively charged residues, whereas the NSI V2 sequences mostly carry polar but uncharged residues at these positions which results in a higher net positive charge for this region in SI genes (Fig. 4). Computer analysis (Garnier-Robson analysis [15]) shows that the variation of residues 8 and 17 greatly affects the predicted secondary structure of the V2 loop. In contrast to the NSI clone-derived V2 region, the SI clonederived sequence of the $\mathrm{N}$-terminal part of the $\mathrm{V} 2$ region is very likely to form a stable $\alpha$-helix (Fig. 4). The importance of these V2 loop features in syncytium formation is currently being tested in site-directed mutagenesis studies. A previous report describing the mapping of a virus-neutralizing antibody to the V2 region also suggested a role for the V2 region in the infection process (14). Our data support this notion and point to a direct role for the V2 region in HIV-1 gp120-gp41-mediated membrane fusion.

Our data suggest that the V2 and V3 regions are mutually independent in controlling syncytium formation: when derived from 16.1 , both regions transfer considerable SI capacity, with that of the V2 region further enhanced by an enhancing region. Similarly, the same two regions derived from 16.2 transfer full SI capacity when the proper enhancing region, which is the same for both, is present. Thus, the V2 and V3 regions are the primary SIRs that functionally interact with the enhancing regions identified here. Syncytium formation can apparently be achieved through at least two different determinants on gp120, and in the SI envelope genes studied here, both are functional. For the V3 region, basic residues at positions 11 or 28 are linked to the SI phenotype, and all SI isolates so far appear to have this configuration (11). However, it is not known whether the SI phenotype of these isolates is mediated solely through the 
TABLE 2. SIRs with the corresponding enhancing regions

\begin{tabular}{ccl}
\hline SIR & $\begin{array}{c}\text { Syncytium size } \\
\text { induced by } \\
\text { SIR }\end{array}$ & $\begin{array}{c}\text { Protein or fragment causing } \\
\text { enhanced SIR function }\end{array}$ \\
\hline 16.1 & & 16.1 gp41 (AX fragment) \\
V2 & ++ & $?$ \\
V3 & +++ & \\
& & 16.2 CA fragment \\
16.2 & $++/+$ & 16.2 CA fragment \\
V2 & $++/+$ & \\
V3 &
\end{tabular}

$\mathrm{V} 3$ region or whether the $\mathrm{V} 2$ region also plays a role. It would be of interest to determine whether a consensus SI configuration for $\mathrm{V} 2$ can be found in order to answer these questions.

For the context-dependent SIRs identified in this study, additional sequences are required for transfer of maximal SI capacity. The transfer of SI capacity by the $16.2 \mathrm{~V} 3$ region is enhanced by simultaneous exchange of at least the CvnIAvrII envelope region at the carboxy terminus of gp120 (constructs 24SA and 23SA [Fig. 3C]). To increase transfer of SI capacity by the V2 region of the same (16.2) SI envelope, this CvnI-AvrII region is apparently necessary (constructs $42 \mathrm{HC}, 42 \mathrm{SSCX}$, and $32 \mathrm{HC}$ ). This fragment contains the V4-C4-V5 regions on gp120 and part of gp41. Interestingly, evidence for physical interaction between the $\mathrm{C} 4$ region and the V3 loop has been described by others (39) and may well explain the observed functional interaction between the V3 as an SIR and this enhancing region.

Transfer of SI capacity by the $16.1 \mathrm{~V} 2$ region is enhanced by the AX region of the same SI gene (construct 31SA). This same $A X$ region from 16.1 , but not 16.2 , could also transfer some SI capacity to NSI envelope 16.3 , probably through activation of a cryptic context-dependent SIR on 16.3. For an overview of the functional interactions between SIRs and enhancing regions, see Table 2.

Identification of any region as an SIR or an enhancing region heavily depends on the sequences of all other regions involved. Probably even the context-independent SIR (V3 region 16.1) functionally interacts with the enhancing regions identified here, but the exact sequence of the enhancing region is less restricted than for the other SIRs. Cryptic SIRs on NSI envelope genes (for instance, 16.3) can be activated by acquisition of an enhancing region with the proper sequence, e.g., through recombination. We have previously demonstrated that recombination does occur in vivo in an infected individual (18). This result means that regions involved in syncytium formation do not necessarily have to coevolve in the same virus to generate an SI variant. Even recombination between two NSI envelope genes, one containing a cryptic SIR and the other containing an enhancing region could therefore lead to an SI virus. We are currently testing this hypothesis. Such mechanisms greatly enhance the dynamics of the evolution of HIV-1.

\section{ACKNOWLEDGMENTS}

We thank Mathijs Tersmette and collaborators for providing the biological HIV-1 clones and Eric Hunter and Susan J. Roberts of the University of Alabama at Birmingham, Birmingham, for giving us the pSRHS expression plasmid. Furthermore, we thank Jan Groen and John P. Moore for valuable advice on the ELISA technique, Heinz Broeders for help in the immunofluorescence assay, Conny W. H. M. Kruyssen and Miek C. Eskens-Jansen for secretarial assistance, and the Medical Research Council for providing reagents.

This work was supported by grants from the Advisory Council on Health Research (RGO 88-75/89039 [A.C.A. and P.L.]).

\section{REFERENCES}

1. Andeweg, A. C., M. Groenink, P. Leeflang, R. E. Y. De Goede, A. D. M. E. Osterhaus, M. Tersmette, and M. L. Bosch. 1992. Genetic and functional analysis of a set of human immunodeficiency virus type 1 envelope genes obtained from biological clones with varying syncytium inducing capacities. AIDS Res. Human Retroviruses 8:1803-1813.

2. Bosch, M. L., P. L. Earl, K. Fargnoli, S. Picciafuoco, F. Giombini, F. Wong-Staal, and G. Franchini. 1989. Identification of the fusion peptide of primate immunodeficiency viruses. Science (Washington, D.C.) 244:694-697.

3. Chakrabarti, S., K. Brechling, and B. Moss. 1985. Vaccinia virus expression vector: coexpression of beta-galactosidase provides visual screening of recombinant virus plaques. Mol. Cell. Biol. 5:3403-3409.

4. Cheng-Mayer, C., D. Seto, M. Tateno, and J. A. Levy. 1988. Biologic features of HIV-1 that correlate with virulence in the host. Science (Washington, D.C.) 240:80-82.

5. Cheng-Mayer, C., T. Shioda, and J. A. Levy. 1991. Host range, replicative, and cytopathic properties of human immunodeficiency virus type 1 are determined by very few amino acid changes in tat and gp120. J. Virol. 65:6931-6941.

6. Dalgleish, A. G., P. C. L. Beverley, P. R. Clapham, D. H. Crawford, M. F. Greaves, and R. A. Weiss. 1984. The CD4 (T4) antigen is an essential component of the receptor for the AIDS retrovirus. Nature (London) 312:763-767.

7. Dalgleish, A. G., T. C. Chanh, R. C. Kennedy, P. Kanda, P. R. Clapham, and R. A. Weiss. 1988. Neutralization of diverse HIV-1 strains by monoclonal antibodies raised against a gp41 synthetic peptide. Virology 165:209-215.

8. de Jong, J. J., J. Goudsmit, W. Keulen, B. Klaver, W. Krone, M. Tersmette, and A. de Ronde. 1992. Human immunodeficiency virus type 1 clones chimeric for the envelope V3 domain differ in syncytium formation and replication capacity. J. Virol. 66: 757-765.

9. Dubay, J. W., S. J. Roberts, B. H. Hahn, and E. H. Hunter. 1992. Truncation of the human immunodeficiency virus type I transmembrane glycoprotein cytoplasmic domain blocks virus infectivity. J. Virol. 66:6616-6625.

10. Fenyö, E. M., L. Morfeldt Månson, F. Chiodi, B. Lind, A. Von Gegerfelt, J. Albert, E. Olausson, and B. Åsjö. 1988. Distinct replicative and cytopathic characteristics of human immunodeficiency virus isolates. J. Virol. 62:4414-4419.

11. Fouchier, R. A. M., M. Groenink, N. A. Kootstra, M. Tersmette, H. G. Huisman, F. Miedema, and H. Schuitemaker. 1992. Phenotype-associated sequence variation in the third variable domain of the human immunodeficiency virus type 1 gp120 molecule. J. Virol. 66:3183-3187.

12. Freed, E. O., D. J. Myers, and R. Risser. 1990. Characterization of the fusion domain of the human immunodeficiency virus type 1 envelope glycoprotein gp41. Proc. Natl. Acad. Sci. USA 87:4650-4654.

13. Freed, E. O., D. J. Myers, and R. Risser. 1991. Identification of the principal neutralizing determinant of human immunodeficiency virus type 1 as a fusion domain. J. Virol. 65:190-194.

14. Fung, M. S. C., C. R. Y. Sun, W. L. Gordon, R.-S. Liou, T. W. Chang, W. N. C. Sun, E. S. Daar, and D. D. Ho. 1992. Identification and characterization of a neutralization site within the second variable region of human immunodeficiency virus type 1 gp120. J. Virol. 66:848-856.

15. Garnier, J., D. Osguthorpe, and B. Robson. 1978. Analysis of the accuracy and implications of simple methods for predicting the secondary structure of globular proteins. J. Mol. Biol. 120:97-120.

16. Goudsmit, J., C. A. B. Boucher, R. H. Meloen, L. G. Epstein, L. Smit, L. van der Hoek, and M. Bakker. 1988. Human antibody response to a strain-specific HIV-1 gp120 epitope associated with cell fusion inhibition. AIDS 2:157-164. 
17. Goudsmit, J., C. Debouck, R. H. Meloen, L. Smit, M. Bakker, D. M. Asher, A. V. Wolfi, C. J. Gibbs, Jr., and D. C. Gajdusek. 1988. Human immunodeficiency virus type 1 neutralization epitope with conserved architecture elicits early type-specific antibodies in experimentally infected chimpanzees. Proc. Natl. Acad. Sci. USA 85:4478-4482.

18. Groenink, M., A. C. Andeweg, R. A. M. Fouchier, S. Broersen, R. C. M. van der Jagt, H. Schuitemaker, R. E. Y. de Goede, M. L. Bosch, H. G. Huisman, and M. Tersmette. 1992. Phenotype-associated env gene variation among eight related human immunodeficiency virus type 1 clones: evidence for in vivo recombination and determinants of cytotropism outside the V3 domain. J. Virol. 66:6175-6180.

19. Helseth, E., U. Olshevsky, D. Gabuzda, B. Ardman, W. Haseltine, and J. Sodroski. 1990. Changes in the transmembrane region of the human immunodeficiency virus type 1 gp41 envelope glycoprotein affect membrane fusion. J. Virol. 64:6314 6318.

20. Hwang, S. S., T. J. Boyle, H. K. Lyerty, and B. R. Cullen. 1991. Identification of the envelope V3 loop as the primary determinant of cell tropism in HIV-1. Science (Washington, D.C.) 253:71-74.

21. Koot, M., A. H. V. Vos, R. P. M. Keet, R. E. Y. de Goede, M. W. Dercksen, F. G. Terpstra, R. A. Coutinho, F. Miedema, and M. Tersmette. 1992. HIV-1 biological phenotype in long-term infected individuals evaluated with an MT-2 cocultivation assay. AIDS 6:49-54.

22. Lifson, J. D., M. B. Feinberg, G. R. Reyes, L. Rabin, B. Banapour, S. Chakrabarti, B. Moss, F. Wong-Staal, K. S. Steimer, and E. G. Engleman. 1986. Induction of CD4-dependent cell fusion by the HTLV-III/LAV envelope glycoprotein. Nature (London) 323:725-728.

23. Lifson, J. D., G. R. Reyes, M. S. McGrath, B. S. Stein, and E. G. Engleman. 1986. AIDS retrovirus induced cytopathology: giant cell formation and involvement of CD4 antigen. Science (Washington, D.C.) 232:1123-1126.

24. Moore, J. P. 1990. Simple methods for monitoring HIV-1 and HIV-2 gp120 binding to soluble CD4 by enzyme-linked immunosorbent assay: HIV-2 has a 25 -fold lower affinity than HIV-1 for soluble CD4. AIDS 4:297-305.

25. Moore, J. P., and R. F. Jarrett. 1988. Sensitive ELISA for the gp120 and gp160 surface glycoproteins of HIV-1. AIDS Res. Human Retroviruses 4:369-380.

26. Olshevsky, U., E. Helseth, C. Furman, J. Li, W. Haseltine, and J. Sodroski. 1990. Identification of individual human immunodeficiency virus type 1 gp120 amino acids important for CD4 receptor binding. J. Virol. 64:5701-5707.

27. Page, K. A., S. M. Stearns, and D. R. Littman. 1992. Analysis of mutations in the V3 domain of gp160 that affect fusion and infectivity. J. Virol. 66:524-533.

28. Palker, T. J., M. E. Clark, A. J. Langlois, T. J. Matthews, K. J. Weinhold, R. R. Randall, D. P. Bolognesi, and B. F. Haynes. 1988. Type-specific neutralization of the human immunodeficiency virus with antibodies to env-encoded synthetic peptides. Proc. Natl. Acad. Sci. USA 85:1932-1936.
29. Popovic, M., M. G. Sarngadharan, E. Read, and R. C. Gallo. 1984. Detection, isolation, and continuous production of cytopathic retroviruses (HTLV-III) from patients with AIDS and pre-AIDS. Science 224:497-500.

30. Robinson, J. E., D. Holton, S. Pacheco-Morell, J. Liu, and H. McMurdo. 1990. Identification of conserved and variant epitopes of human immunodeficiency virus type 1 (HIV-1) gp120 by human monoclonal antibodies produced by EBVtransformed cell lines. AIDS Res. Human Retroviruses 6:567579.

31. Salter, R. D., D. N. Howell, and P. Cresswell. 1985. Genes regulating HLA class I antigen expression in T-B lymphoblast hybrids. Immunogenetics 21:235-246.

32. Schutten, M., A. McKnight, R. C. Huisman, M. Thali, J. A. McKeating, J. Sodroski, J. Goudsmit, and A. D. M. E. Osterhaus. Further characterization of an antigenic site of HIV-1 gp120 recognized by virus neutralizing human monoclonal antibodies. Submitted for publication.

33. Smith, S. D., M. Shatsky, P. S. Cohen, R. Warnke, M. P. Link, and B. E. Glader. 1984. Monoclonal antibody and enzymatic profiles of human malignant T-lymphoid cells and derived cell lines. Cancer Res. 44:5657-5660.

34. Sodroski, J., W. G. Goh, C. Rosen, K. Campbell, and W. A. Haseltine. 1986. Role of the HTLV-III/LAV envelope in syncytium formation and cytopathicity. Nature (London) 322:470474.

35. Tersmette, M., R. E. Y. De Goede, B. J. M. Al, I. N. Winkel, R. A. Gruters, H. T. Cuypers, H. G. Huisman, and F. Miedema. 1988. Differential syncytium-inducing capacity of human immunodeficiency virus isolates: frequent detection of syncytiuminducing isolates in patients with acquired immunodeficiency syndrome (AIDS) and AIDS-related complex. J. Virol. 62:20262032.

36. Tersmette, M., J. M. A. Lange, R. E. Y. De Goede, F. de Wolf, J. K. M. Eeftink Schattenkerk, P. T. A. Schellekens, R. A. Coutinho, J. G. Huisman, J. Goudsmit, and F. Miedema. 1989. Association between biological properties of human immunodeficiency virus variants and risk for AIDS and AIDS mortality. Lancet i:983-988.

37. Thali, M., U. Olshevsky, C. Furman, D. Gabuzda, M. Posner, and J. Sodroski. 1991. Characterization of a discontinuous human immunodeficiency virus type 1 gp120 epitope recognized by a broadly reactive neutralizing human monoclonal antibody. J. Virol. 65:6188-6193.

38. Willey, R. L., D. H. Smith, L. A. Lasky, T. S. Theodore, P. L. Earl, B. Moss, D. J. Capon, and M. A. Martin. 1988. In vitro mutagenesis identifies a region within the envelope gene of the human immunodeficiency virus that is critical for infectivity. $J$. Virol. 62:139-147.

39. Wyatt, R., M. Thali, S. Tilley, A. Pinter, M. Posner, D. Ho, J. Robinson, and J. Sodroski. 1992. Relationship of the human immunodeficiency virus type $1 \mathrm{gp} 120$ third variable loop to a component of the CD4 binding site in the fourth conserved region. J. Virol. 66:6997-7004. 